\title{
Novel Study on Improve the Image Quality using Gradient Profile Sharpness for High Resolution Images
}

\author{
B.V. Harika, A. Monika, G. Sowjanya, G. \\ Vijay Kumar \\ UG Scholar, \\ Department of Computer Science \& \\ Engineering \\ St.Ann's College of Engineering \& Technology, \\ Chirala, A.P, India.
}

\author{
A. Veeraswamy, PhD \\ Associate Professor, \\ Department of Computer Science \& Engineering, \\ St.Ann's College of Engineering \& Technology, \\ Chirala, A.P, India.
}

\begin{abstract}
Single image super resolution is a classic and active image processing problem, which aims to generate high resolution (HR) image from a low-resolution input image. Due to the severely under-determined nature of this problem, an effective image prior is necessary to make the problem solvable, and to improve the quality of generated images. In this paper, we proposed a novel image super resolution algorithm is based on gradient profile sharpness (GPS). GPS is an edge sharpness metric, which is extracted from two gradient description models that is a triangle model and a Gaussian mixture model for the description of different kinds of gradient profiles. Then, the transformation relationship of GPSs in different image resolutions is studied statistically, and the parameter of the relationship is estimated automatically. Based on the estimated GPS transformation relationship, two gradient profile transformation models are proposed for two profile description models, which can keep profile shape and profile gradient magnitude sum consistent during profile transformation. Finally, the target gradient field of HR image is generated from the transformed gradient profiles is added as the image prior in HR image reconstruction model. Extensive experiments are conducted to evaluate the proposed algorithm in subjective visual effect, objective quality, and computation time. The experimental results demonstrate that the proposed approach can generate superior HR images with better visual quality, lower reconstruction error, and acceptable computation efficiency as compared with state-of-the-art works. We compared before applying the GPS and also after apply the GPS and for an image the results shows the after applying GPS give high resolution.
\end{abstract}

\section{Keywords \\ GGD, GPS, ICBI}

\section{INTRODUCTION}

The goal of single image super-resolution is to construct a high resolution (HR) image from a low resolution (LR) image input. This problem is a classical and active topic in image processing, which is also a crucial step in many practical situations, e.g. image display, remote sensing, and medical imaging and so on. However, image super-resolution problem is an inherently ill-posed problem, where many HR images may produce the same LR image when down-sampled. As a result, how to generate an HR image with good visual perception and as similar as its ground truth has become the goal of image super-resolution.

There has been many research works in this field in recent years, which can be mainly classified into three categories those are interpolation-based approaches, learning-based approaches and reconstruction-based approaches. The interpolation-based approaches are the basic image superresolution methods, where currently the bi-linear interpolation and bicubic interpolation are still very popular in practice. More sophisticated interpolation models have also been proposed, e.g. auto-regression model, multi-surface fitting model, edge directed models, sparse representation models and ICBI (Interactive Curvature Based Interpolation) algorithm. Interpolation-based approaches always have fast computation speed. However, as pointed out in, interpolationbased approaches tend to blur high frequency details if the upscaling ratio is large and if the low-resolution image is generated with anti-aliasing operation[7].

The learning-based approaches assume that the lost high frequency details in LR images can be retrieved and hallucinated from a dictionary of image patch pairs. One category of learning-based approaches learns example patches from a data set retrieved from fixed HR image set or website. Naturally, the performance of these approaches is highly correlated to the similarity between the LR image patch and the learned HR image patches. To reduce the dependence on the training HR image, self-example based approaches were proposed, which utilized the observation that patches tended to redundantly recur inside an image within the same image scale as well as across different scales or there existed a transformation relationship across image space[9]. These approaches are more robust, however there are always some artifacts on their super resolution results. Generally, the computational complexity of learning based super resolution approaches is quite high.

To make a tradeoff between algorithm performance and algorithm computational efficiency, many reconstructionbased approaches have been proposed over the years. The reconstruction-based approaches enforce a constraint that the smoothed and down-sampled version of the estimated HR image should be consistent with its LR image. Based on this idea, reconstruction models are proposed using backprojection or convex projection. To make the ill-posed reconstruction problem solvable and to find the best estimated HR image, an effective regularization term should be added as the model constraint[5], which is crucial for the reconstruction-based approaches. Various regularization terms have been proposed based on local gradient enhancement and global gradient spars. Recently, metrics of edge sharpness have attracted researcher's attention as the regularization term, since edges are of primary importance in visual image quality. Fatal introduced the conception of gradient profile to 
describe the spatial resolution of edges, where a gradient profile is represented using three features, i.e. total intensity change, distance from center pixel to the pixel of zero gradient magnitude, and profile standard deviation. Sun et.al [2]. Modeled the normalized gradient profile with a GGD (generalized Gaussian distribution) model, and adopted the standard deviation of GGD model as the edge sharpness metric. With the help of GGD model, methods of could describe gradient profile shapes using an adjustable and controllable model, which played an important role in gradient profile description and transformation. However, the GGD model is symmetric and regularly shaped, which is hard to represent the gradient profiles with asymmetric and complicated shapes. Furthermore, the metric of is extracted from a normalized gradient profile, where the contrast difference between two gradient profiles are neglected in the profile normalization step.

According to the analysis in edge contrast is a key feature for describing edge visual significance, which should be adopted for the computation of edge sharpness. In this paper, a novel edge sharpness metric GPS (gradient profile sharpness) is extracted as the eccentricity of gradient profile description models, which considers both the gradient magnitude and the spatial scattering of a gradient profile.To precisely describe different kinds of gradient profile shapes, a triangle model and a mixed Gaussian model are proposed for short gradient profiles and heavy-tailed gradient profiles respectively [6]. Then the pairs of GPS values under different image resolutions are studied statistically, and a linear GPS transformation relationship is formulated, whose parameter can be estimated automatically in each super-resolution application. Based on the transformed GPS, two gradient profile transformation models are proposed, which can well keep profile shape and profile gradient magnitude sum consistent during the profile transformation. Finally, the target gradient field of HR (high resolution) image is generated from transformed gradient profiles, which is added as the image priors in HR image reconstruction model. Extensive experiments are conducted to fully evaluate the proposed super-resolution approach. It is demonstrated that the proposed approach can generate superior HR images with better visual similarity and lower reconstruction error as compared with state-of-art works.

\section{PROPOSED MODEL}

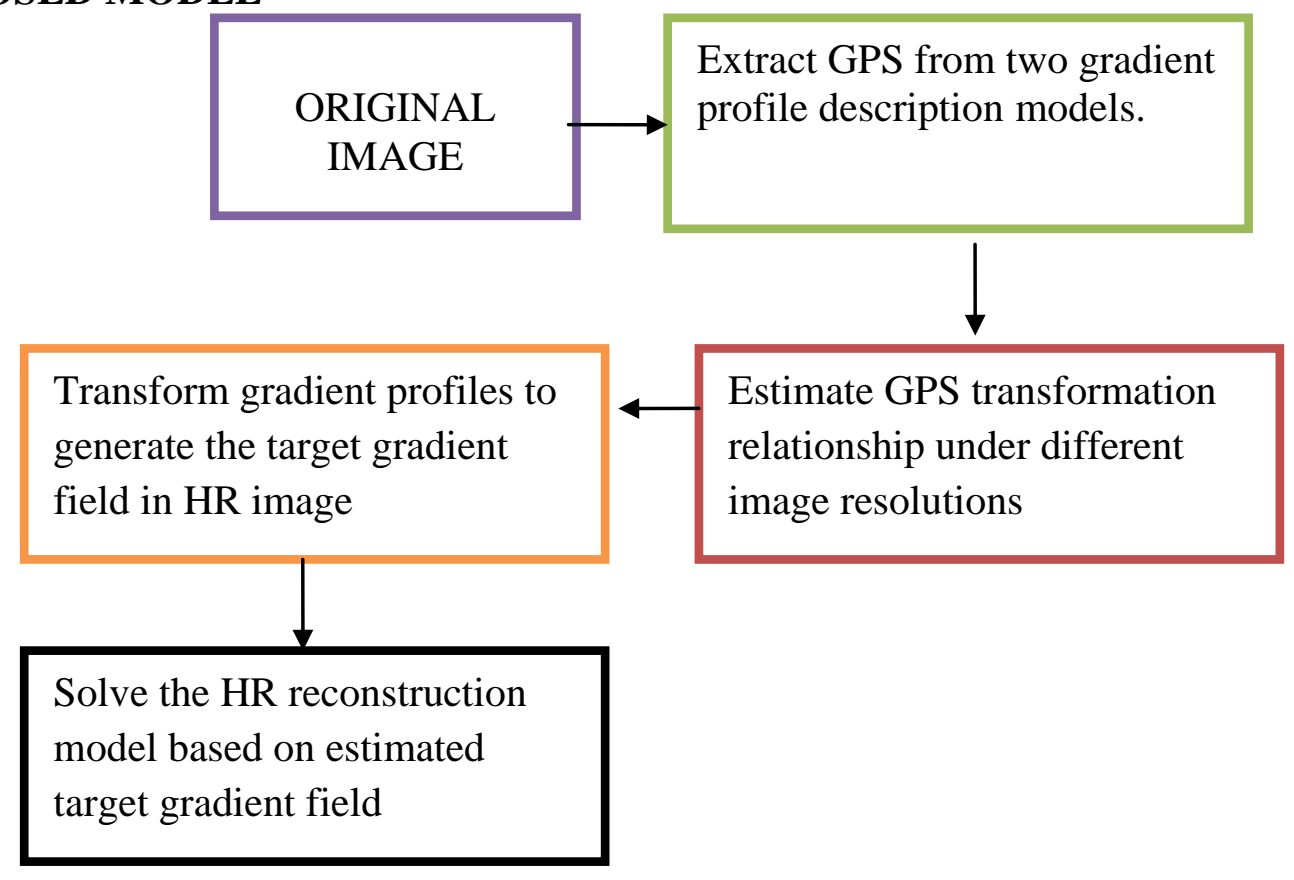

\section{A. Triangle Model}

When edges are sharp or unnoticeable with small intensity changes, the extracted gradient profiles are always short with no tails. For this kind of gradient profiles, a triangle model is most suitable for the profile description[1].

\section{B. Mixed Gaussian Model}

When edges are smooth, gradient profiles become longer and profile shapes become complicated with heavy tails. For such kind of gradient profiles, a mixed Gaussian model is proposed, which is a mixture of two Gaussian models.

\section{Evaluation of Gradient Profile Fitting Performance}

To evaluate the fitting performance of proposed gradient profile description models, an image set containing 10 images from dataset is UCI Repository adopted for gradient profile extraction. For each image, edges are detected using canny algorithm. Then, 1000 edge pixels are selected randomly to produce 10 gradient profiles. For each gradient profile, both the GGD model and the proposed gradient profile description models are adopted in the fitting process. 


\section{RESULTS \& DISSCUSSIONS}

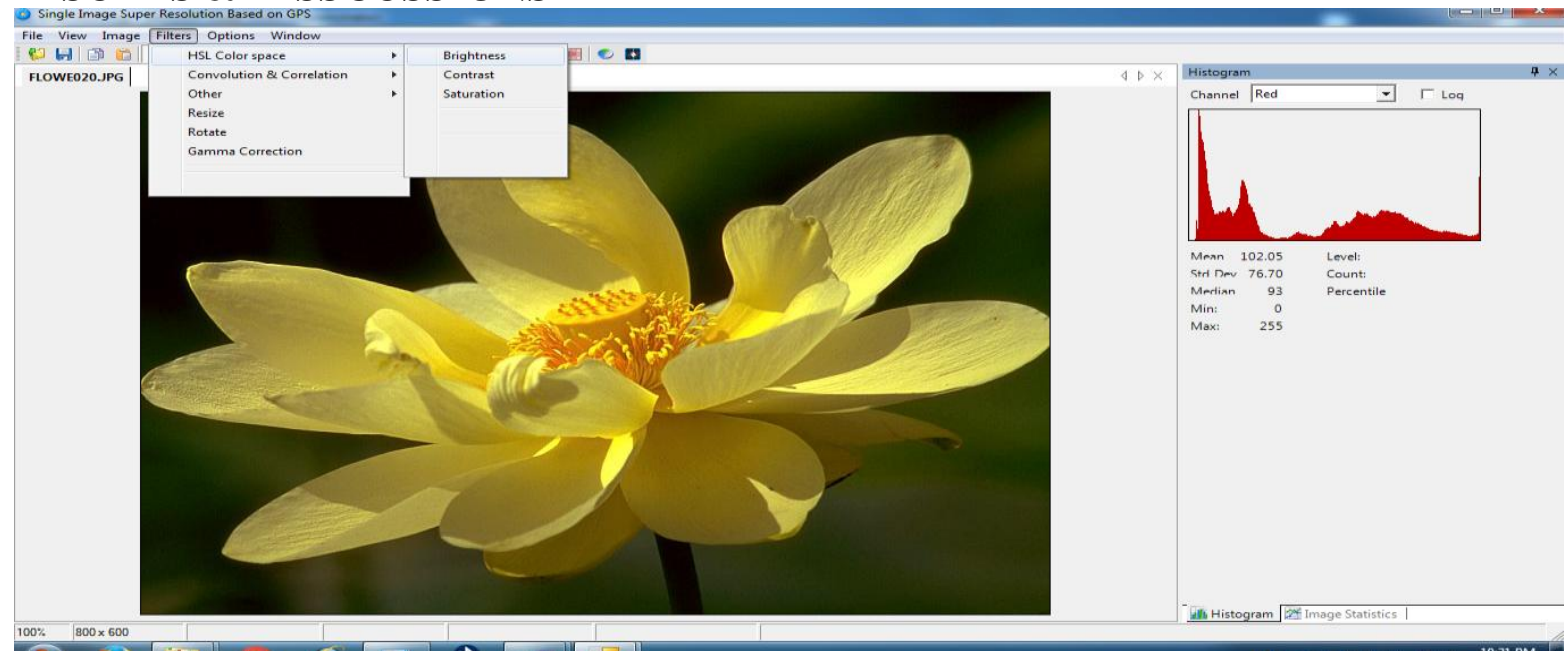

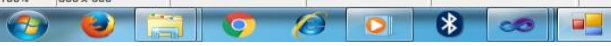

Figure 1:Applying HSL color space i.e.,Brightness of an image

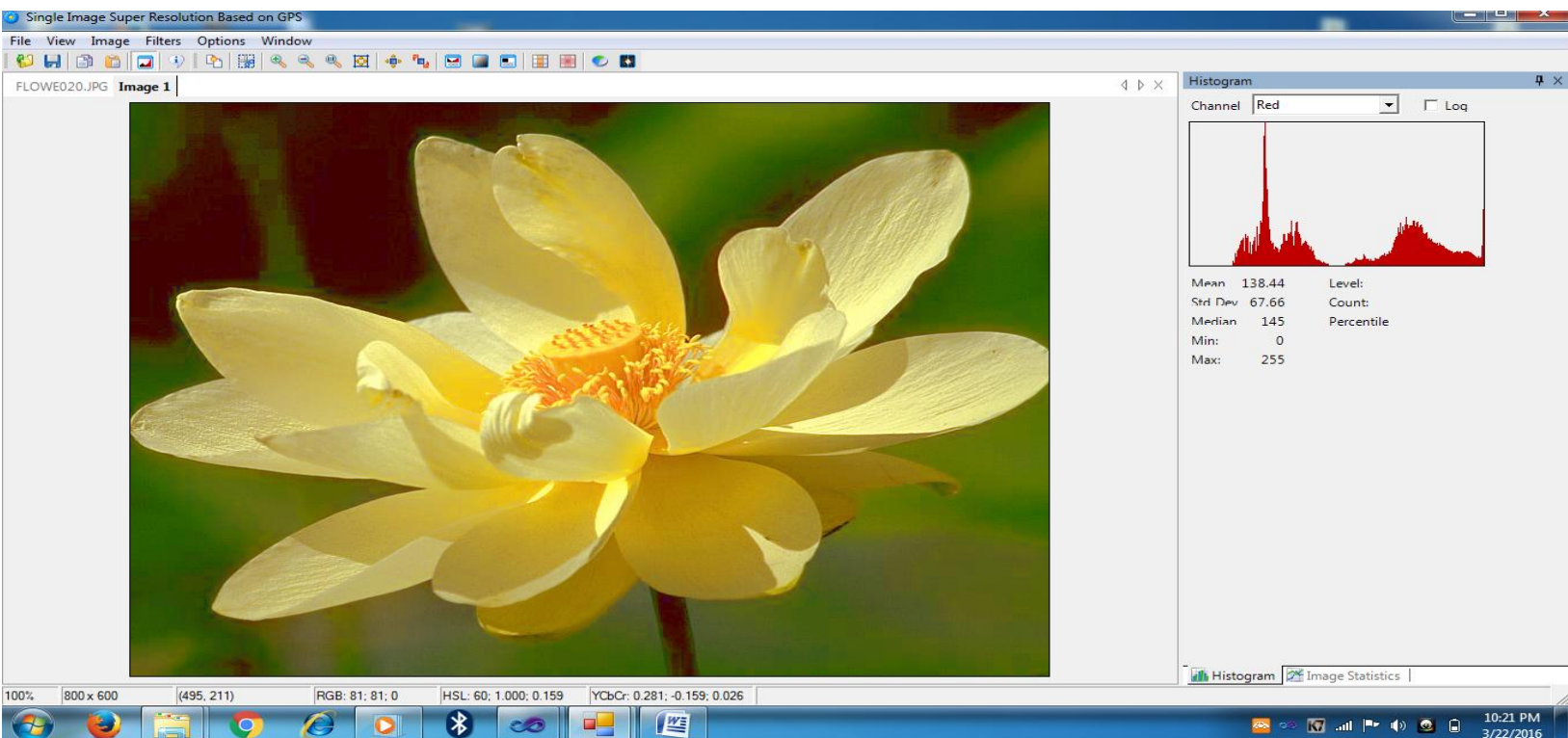

Figure 2:After applying Brightness of an image

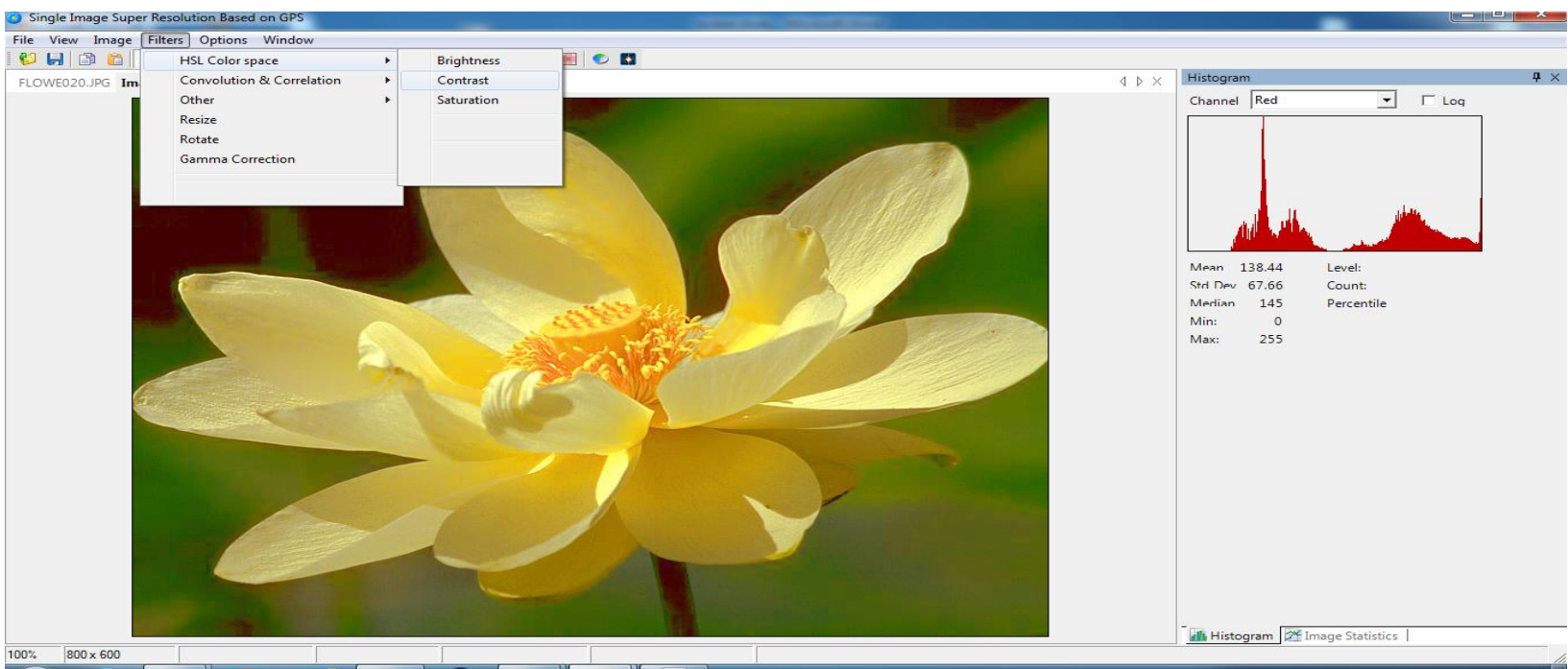




\section{Figure 3:Appying contrast to the image}

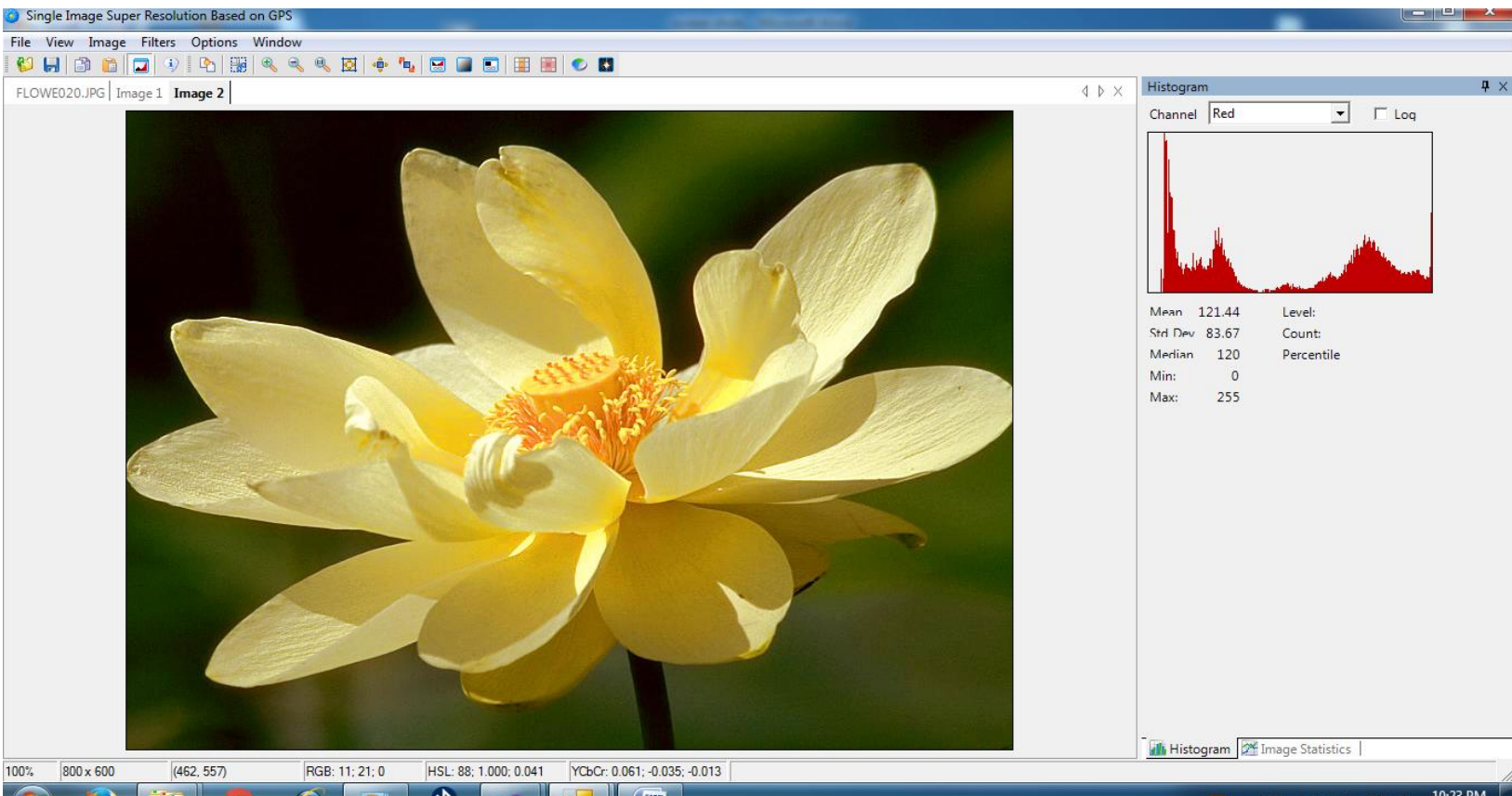

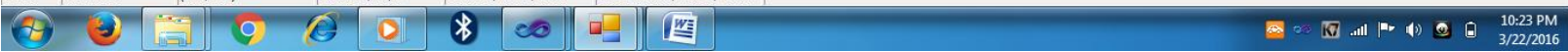

Figure 4:After appying contrast of an image

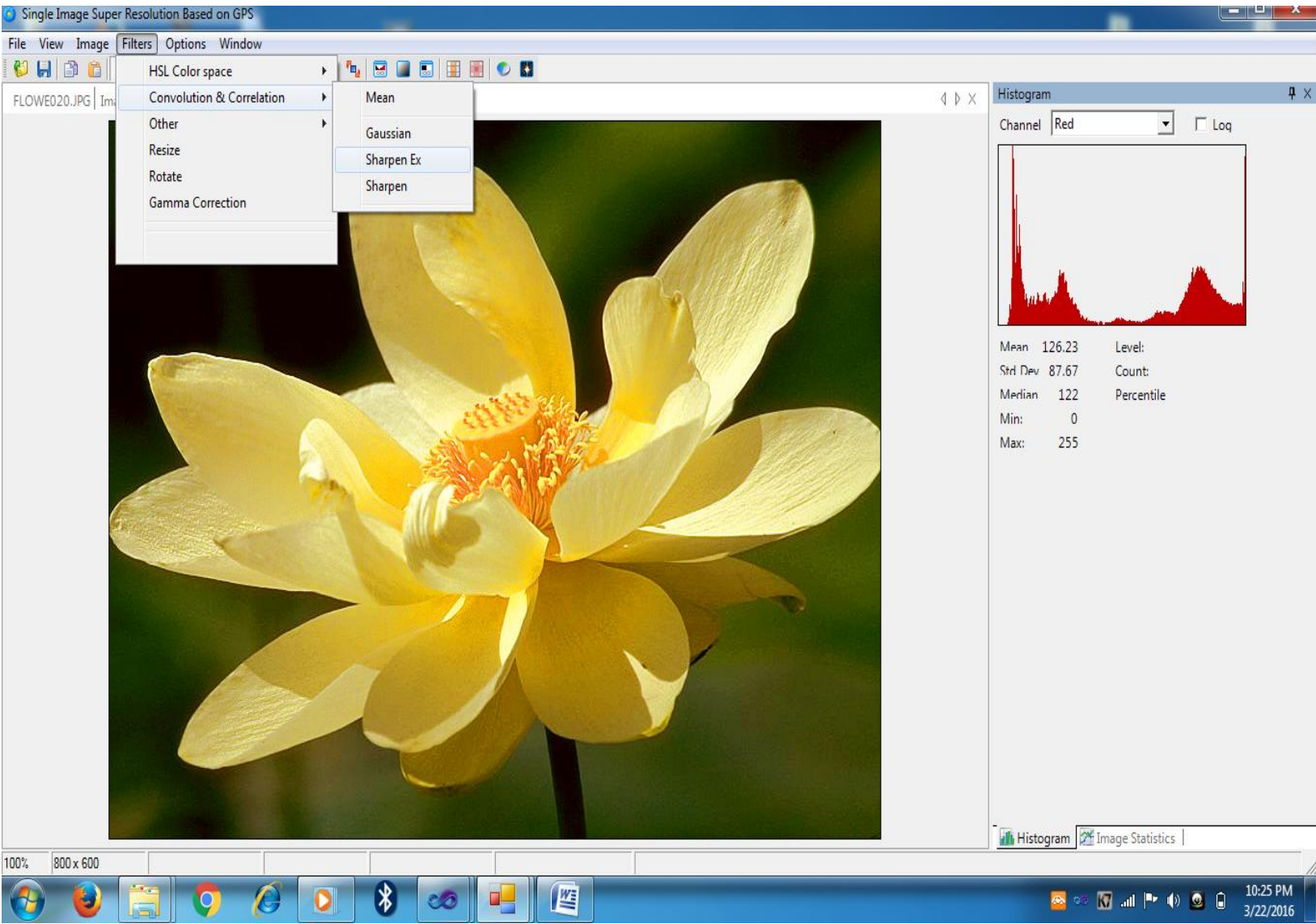

Figure 5:Applying sharpen ex to the image 


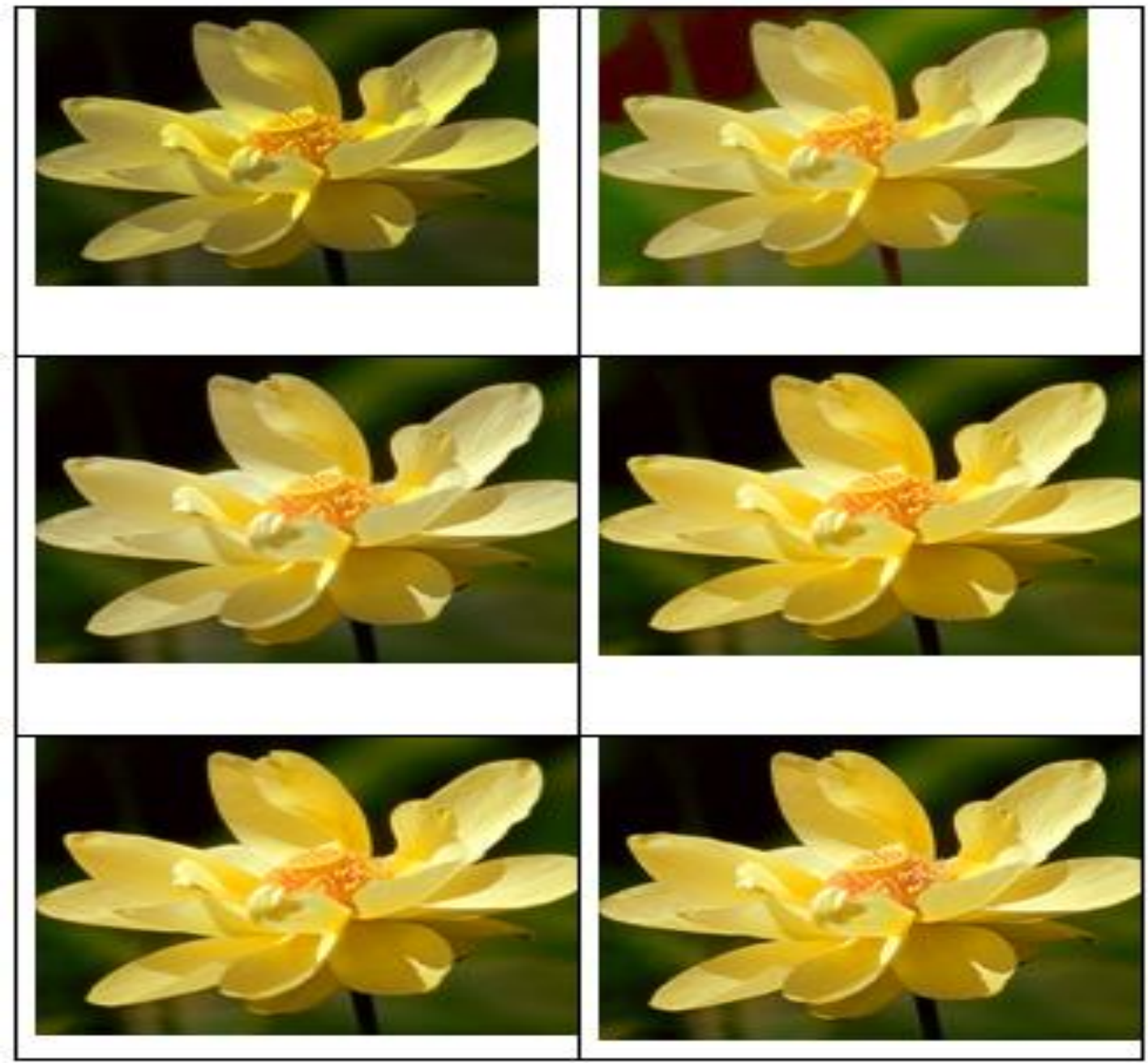

Figure 6:Applying all other filters to the image

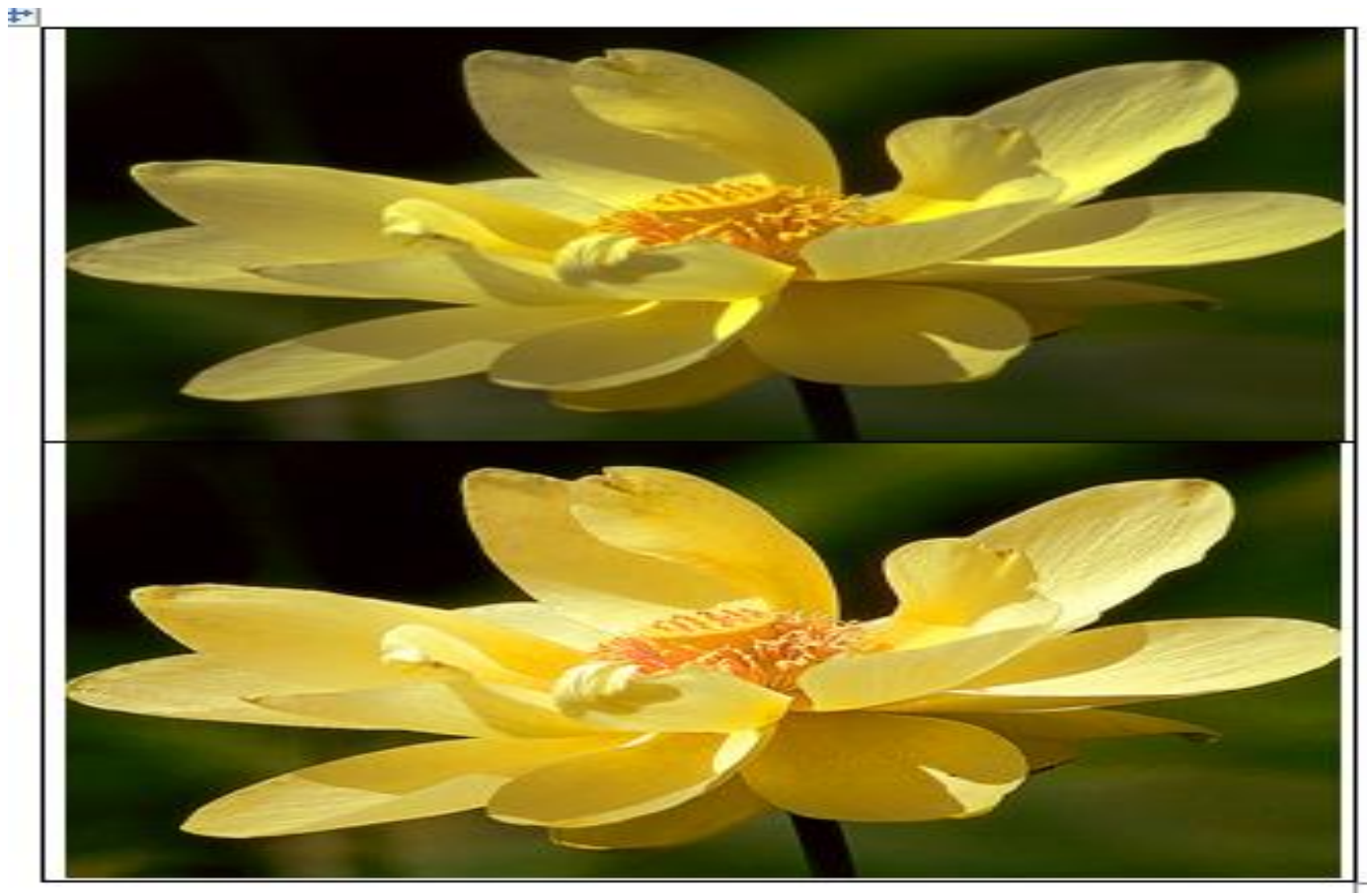

Figure 7:Differences between original image and modified image 


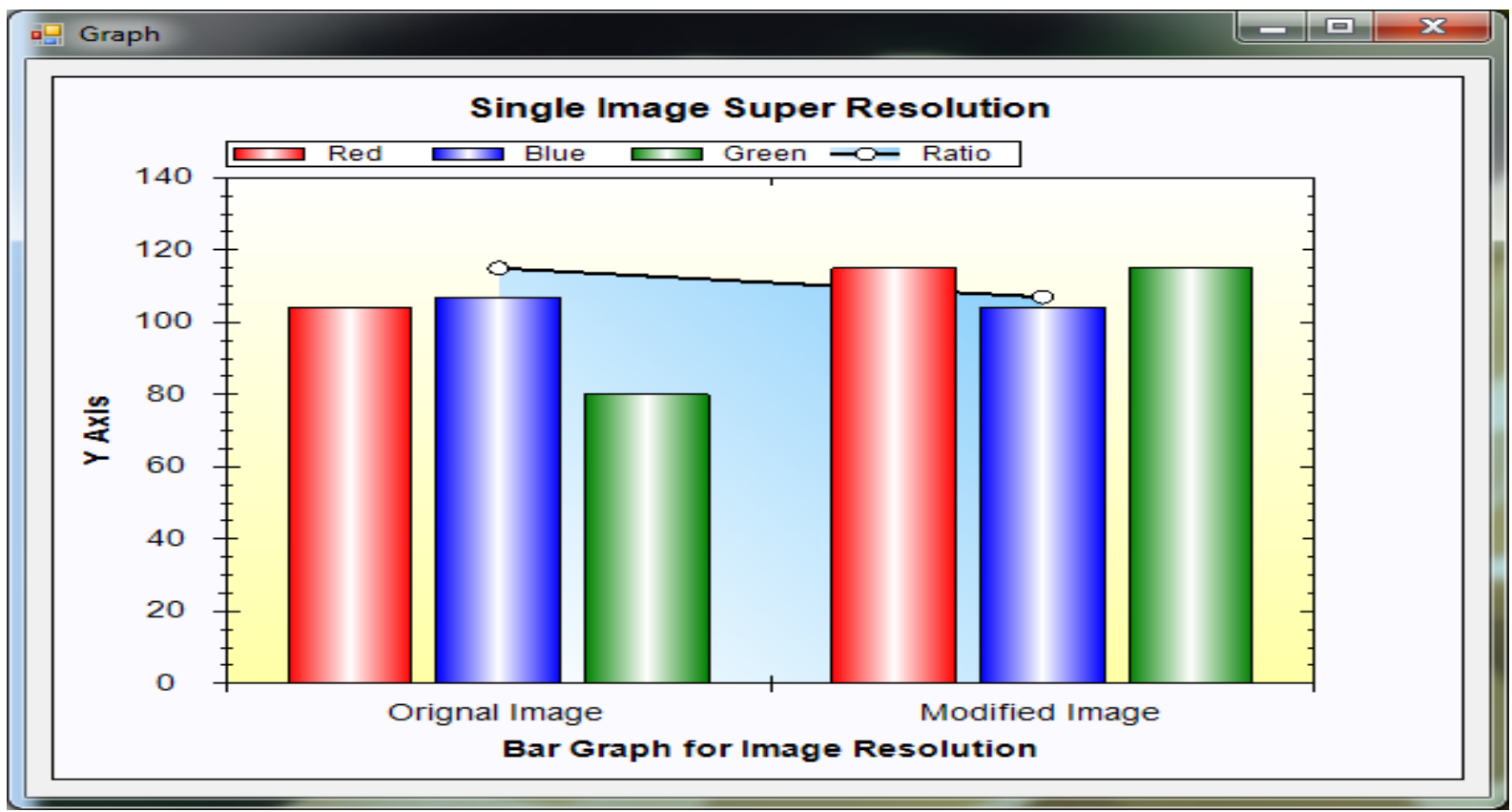

Figure 8: Performance Graph among Original Image and Modified Image

\section{CONCLUSION \& FUTURE WORK}

The proposed system introduced a Single Image Super resolution based on Gradient Profile Sharpness which is enhanced by using DWT-Based Adaptive Edge Map. The two gradient profile description models are introduced for representing gradient profiles with different lengths and different complicated shapes. After the completion of GPS transformation relationship computation process, the Parameter of GPS transformation relationships is estimated automatically. The transformed gradients are utilized as priors in the high resolution image reconstruction. By using DWTBased Adaptive Edge Map is used to remove the artifacts efficiently. The experimental results shows that the proposed system having high accuracy in my future work.

\section{REFERENCES}

[1] H. S. Hou and H. Andrews, "Cubic splines for image interpolation and digital filtering," IEEE Trans. Acoust., Speech Signal Process., vol. 26, no. 6, pp. 508-517, Dec. 1978.

[2] X. Zhang and X. Wu, "Image interpolation by adaptive 2D autoregressive modeling and soft decision estimation," IEEE Trans. Image Process., vol. 17, no. 6, pp. 887-896, Jun. 2008.

[3] F. Zhou, W. Yang, and Q. Liao, "Interpolation-based image super resolution using multisurface fitting," IEEE Trans. Image Process.,vol. 21, no. 7, pp. 3312-3318, Jul. 2012.
[4] S. Mallat and G. Yu, "Super-resolution with sparse mixing estimators,"IEEE Trans. Image Process., vol. 19, no. 11, pp. 2889-2900, Nov. 2010.

[5] W. Dong, L. Zhang, R. Lukac, and G. Shi, "Sparse representation based image interpolation with nonlocal autoregressive modeling," IEEE Trans.Image Process., vol. 22, no. 4, pp. 1382-1394, Apr. 2013.

[6] A. Giachetti and N. Asuni, "Real-time artifact-free image upscaling,"IEEE Trans. Image Process., vol. 20, no. 10, pp. 2760-2768, Oct. 2011.

[7] W. T. Freeman, T. R. Jones, and E. C. Pasztor, "Example-based super-resolution, "IEEE Comput. Graph. Appl., vol. 22, no. 2, pp. 56-65, Mar./Apr. 2002.

[8] J. Yang, J. Wright, T. Huang, and Y. Ma, "Image superresolution as sparse representation of raw image patches," in Proc. IEEE Conf. Comput. Vis. Pattern Recognit., Jun. 2008, pp. 1-8.

[9] K. Zhang, X. Gao, D. Tao, and X. Li, "Multi-scale dictionary for single image super resolution," in Proc. IEEE Conf. Comput. Vis. Pattern Recognit., Jun. 2012, pp. 1114-1121.

[10] S. Yang, M. Wang, Y. Chen, and Y. Sun, "Single-image super-resolution reconstruction via learned geometric dictionaries and clustered sparse coding," IEEE Trans. Image Process., vol. 21, no. 9, pp. 4016-4028, Sep. 2012. 